\title{
Lymphocytes from the site of disease but not blood lymphocytes indicate the cause of arthritis
}

\author{
DENYS K FORD, DOREEN M DA ROZA, AND MICHAEL SCHULZER \\ From the Department of Medicine, University of British Columbia, Canada
}

SUMMARY The $\left[{ }^{3} \mathrm{H}\right]$ thymidine uptake procedure for measuring lymphocyte responses was applied to lymphocytes derived concurrently from synovial effusions and from peripheral blood. The stimulating antigens were crude preparations of those micro-organisms that are related to the enteritis and the non-gonococcal urethritis that precipitate reactive arthritis. Salmonella, shigella, and campylobacter antigens stimulated synovial but not peripheral blood lymphocytes in eight cases of enteric reactive arthritis. Ureaplasma or chlamydia antigens, or both, stimulated synovial lymphocytes in all 12 cases of sexually transmitted reactive arthritis, whereas peripheral blood lymphocytes were only stimulated in four of the 12 cases. In 14 cases of rheumatoid arthritis reactions to either enteric or ureaplasma/chlamydia antigens were minimal from either synovial or peripheral blood lymphocytes. It is concluded that synovial rather than peripheral blood lymphocytes indicate the microbiological cause of reactive arthritis and that similar studies of lymphocytes from the site of local disease might be productive in other diseases.

Key words: synovial lymphocyte responses, $\left[{ }^{3} \mathrm{H}\right]$ thymidine uptake, reactive arthritis, Reiter's syndrome, campylobacter antigen, Enterobacteriaceae antigens, chlamydia/ureaplasma antigens.

Reactive arthritis is a well recognised sequel to
enteric infection with shigella, salmonella,
yersinia, ${ }^{3}$ or campylobacter ${ }^{4}$ organisms. Reactive
arthritis also follows sexually acquired non-
gonococcal urethritis and, though there is still
argument over the cause of non-gonococcal urethri-
tis, both chlamydias and ureaplasmas are generally
accepted as being probably responsible for the
majority of cases. 5 Despite much research effort
there is no clear understanding of the pathogenesis
of these reactive arthritides, though it is evident that
a genetic predisposition is recognisable from the
presence of the HLA-B27 histocompatibility anti-
gen.

Although the enteric and genitourinary pathogens listed above are normally isolated from the stools or urethral exudate when cultures are performed under optimal conditions, these agents are not normally found in the affected joints. Moreover, up to the present, serum antibody studies have not provided significant understanding of the pathogenetic mech-

Accepted for publication 19 March 1985.

Correspondence to Dr Denys K Ford. The Arthritis Centre. 895 West 10th Avenuc, Vancouver, British Columbia. Canada V5Z 1 L7. anisms relating the intestinal or genital infection to the development of arthritis.

In 1979 it was observed that synovial lymphocytes from cases of sexually transmitted reactive arthritis were specifically stimulated by ureaplasma or chlamydia antigens, ${ }^{6}$ and subsequently it was found that Enterobacteriaceae antigens stimulated synovial lymphocytes from cases of enteric reactive arthritis. ${ }^{3}$ The specificity of these reactions was the subject of a later report." This present paper compares the synovial lymphocyte responses with concurrent peripheral blood lymphocyte responses to the relevant microbiological antigens. The validity of this approach seems to have been confirmed by the observations recently reported that synovial. but not peripheral blood lymphocytes, responded to antigen derived from the causative spirochaete in cases of Lyme arthritis."

\section{Materials and methods}

PATIENTS

All patients for this study had concurrent observations on both synovial and peripheral blood lymphocytes. 


\section{Enteric cases}

Five patients were studied in whom arthritis followed an enteric infection that was bacteriologically defined. In four of these the causative organism was cultured from the stools. In the other patient a diagnosis of Shigella flexneri 2 enteritis was made from a 1/64 serum antibody titre, but no antibodies were shown against Salmonella typhi, Salm. paratyphi $B$, and Yersinia enterocolitica; this patient had contracted diarrhoea in Mexico, and stool cultures were obtained only after the diarrhoea had subsided, when no pathogens were found.

In three additional patients an attack of diarrhoea of undiagnosed aetiology was followed by an episode of arthritis. None of these had previous arthritis, and in each the arthritis was clinically attributed to the diarrhoea.

The clinical data on these eight patients are shown in Table 1. Two patients had arthritis confined to one knee and one patient to both knees; the others had arthritis of between three and five joints. In three of the patients there was an associated conjunctivitis. All the patients were improving within two months of onset of their arthritis, and the clinical course of their arthritis was typical fo $\$$ enteric reactive arthritis.

Sexually transmitted cases

Twelve patients had arthritis in association with sexually transmitted urethritis, and the clinical date on these subjects are also shown in Table 1. The aetiology of the urethritis was not defined in thes subjects because the majority of patients seen for reactive arthritis no longer have active urethritis owing to the interval from onset of the urethritis t $\vec{\Phi}$ the time of rheumatological contact, or because they have already been treated with antibiotige by the time they are evaluated rheumatologically Four of the 12 patients had previous episodes of reactive arthritis, and one patient had an associate conjuctivitis. The exact temporal relationshig between the urethritis and the arthritis was indefinite in two cases, patients 14 and 18 though both patients described urethral discharge and arthritis as occurring at about the same time The course of the arthritis in these 12 cases conformed to that of sexually transmittes reactive arthritis.

Table 1 Clinical data on eight patients with enteric reactive arthritis and 12 patients with sexually transmitted reace arthritis

\begin{tabular}{lllll}
\hline $\begin{array}{l}\text { Patient Sex } \\
\text { No }\end{array}$ & Age & $\begin{array}{l}\text { Previous } \\
\text { arthritis }\end{array}$ & Conjunctivitis & Precipitating event
\end{tabular}

No arthritis

\begin{tabular}{|c|c|c|c|c|c|c|c|}
\hline & & & & & & $\begin{array}{l}\text { arthritis } \\
\text { (weeks) }\end{array}$ & lwee \\
\hline 1 & M & 28 & 0 & 0 & Salm. typhimurium enteritis & 2 & 2 \\
\hline 2 & M & 65 & 0 & 0 & Salm. typhimurium enteritis & 2 & 2 \\
\hline 3 & M & 42 & 0 & + & Salmonella B enteritis & 1 & 2 \\
\hline 4 & $\mathbf{M}$ & 23 & 0 & + & Sh. flexneri enteritis & 4 & 1 \\
\hline 5 & M & 33 & 0 & + & Campylobacter jejuni enteritis & 1 & 1 \\
\hline 6 & $\mathrm{~F}$ & 37 & 0 & 0 & Diarrhoca & 2 & 1 \\
\hline 7 & M & 27 & 0 & 0 & Diarrhoca & 1 & 4 \\
\hline 8 & M & 25 & 0 & 0 & Diarrhoca & 3 & 4 \\
\hline 9 & M & 35 & 0 & 0 & Urethritis & 1 & 1 \\
\hline 10 & M & 19 & 0 & 0 & Urethritis & 2 & 1 \\
\hline 11 & M & 21 & 0 & 0 & Urethritis & 2 & 2 \\
\hline 12 & M & 19 & $\begin{array}{l}\text { Reiter's syndrome } \\
\text { eight months before }\end{array}$ & 0 & Urethritis & 1 & 3 \\
\hline 13 & M & 24 & $\begin{array}{l}\text { Yersinia arthritis } \\
\text { five years before }\end{array}$ & 0 & Urethritis & 1 & 1 \\
\hline 14 & $\mathbf{M}$ & 32 & $\begin{array}{l}\text { Reiter's syndrome } \\
\text { one year before }\end{array}$ & 0 & Urethritis & $?$ & 6 \\
\hline 15 & $\mathbf{M}$ & 32 & 0 & 0 & Urethritis & 1 & 1 \\
\hline 16 & M & 18 & $\begin{array}{l}\text { Urethritis and } \\
\text { arthritis three }\end{array}$ & & & & \\
\hline & & & years before & 0 & Urethritis & 1 & 2 \\
\hline 17 & M & 20 & 0 & 0 & Urethritis & 2 & 2 \\
\hline 18 & M & 42 & 0 & 0 & Urethritis & $?$ & 12 \\
\hline 19 & $\mathbf{M}$ & 24 & 0 & 0 & Urethritis & 2 & 9 \\
\hline 20 & $\mathbf{M}$ & 30 & 0 & + & Urethritis & 1 & 6 \\
\hline
\end{tabular}


Rheumatoid arthritis cases

Fourteen patients with 'definite rheumatoid arthritis' by the criteria of the American Rheumatism Association were studied. Seven of the 14 patients were male, in contrast to 19 of the 20 patients with reactive arthritis. The average age of the seven male patients was 46 years, which compares with an average of 35 years for the patients with enteric reactive arthritis and an average of 26 years for the patients with sexually transmitted reactive arthritis. The responses of the blood and synovial lymphocytes to antigenic stimulation in the rheumatoid subjects were not affected by age or sex, and consequently, the data from all the cases have been included.

\section{LABORATORY METHODS}

\section{$\left[{ }^{3} \mathrm{H}\right]$ Thymidine uptake procedures}

Blood and synovial fluid samples were collected in sterile Vacutainer tubes (Becton Dickinson) containing heparin. Blood plasma with white cells was obtained by gravity sedimentation and the cell deposit from synovial fluid by centrifugation after dilution with three to four volumes of RPMI 1640 medium (Gibco). Ficoll-Hypaque (Pharmacia) separated mononuclear cells were cultured in RPMI 1640 medium supplemented with $10 \%$ human heparinised $\mathrm{AB}$ plasma (Red Cross) and with penicillin (100 units $/ \mathrm{ml})$ and streptomycin (100 $\mathrm{mg} / \mathrm{l}$ ). Microtitre plates (Falcon) were employed for all experiments with 50000 lymphocytes per well, as defined by crystal violet staining. The appropriate antigen, with triplicate wells for each dilution, was added on day 0 and the plates incubated at $37^{\circ} \mathrm{C}$ in $5 \% \mathrm{CO}_{2}$ for seven days. On the day of harvest $\left[{ }^{3} \mathrm{H}\right]$ thymidine (New England Nuclear) at $2 \mu \mathrm{Ci} /$ well was added for four to six hours, and the cells were harvested onto filter discs by standard harvesting procedures. Scintillation counting was performed with $6 \mathrm{ml}$ of toluene-Omnifluor (New England Nuclear) and a Searle Isocap 300 counter. The antigen response was defined as the mean of the counts of the triplicate cultures giving the maximum response to any one of the three antigen dilutions. The results were expressed as a stimulation index (SI), this being the ratio of cpm in the maximally stimulated cultures to the cpm of unstimulated cultures.

\section{Antigens}

From the inception of the studies it was considered that crude antigenic preparations should be employed because methods of purification might result in the loss of a relevant antigen. It was recognised from the start that the use of crude antigens might complicate the interpretation of responses from the presence of common antigens or reaction to endotoxin. An additional policy decision was that antigens would not be standardised by the usual chemical or opacity procedures but standardised by comparative stimulatory capacity with previously employed antigenic batches. In the initial standardisation and evaluation of antigens five to six serial twofold dilutions were tested, but in the later routine procedure of testing lymphocytes only three serial twofold dilutions were employed for reasons of efficiency, usually $1: 8,1: 16$, and $1: 32$. The antigen dilution was that added in a $0.05 \mathrm{ml}$ volume to the lymphocyte cultures with a $0.25 \mathrm{ml}$ final volume in each microtitre well.

Ureaplasma antigen. $U$. urealyticum serotype $5^{10}$ antigen was obtained from $500 \mathrm{ml} 24 \mathrm{~h}$ cultures in Difco mycoplasma broth supplemented with $10 \%$ frozen Microbiological Associates yeast extract, 5\% horse serum, $0.05 \%$ urea, and $0.002 \%$ phenol red. The organisms were deposited by centrifugation at $45000 \mathrm{~g}$ for $15 \mathrm{~min}$, and the deposit washed three times in phosphate-buffered saline (PBS). The antigen was resuspended in $5 \mathrm{ml}$ distilled water, frozen and thawed three times, and stored at $-70^{\circ} \mathrm{C}$ in aliquots. Initially in the studies an uninoculated mycoplasma broth control antigen was employed, but this did not stimulate lymphocytes and therefore its use was discontinued. Previous studies have shown that these lymphocyte responses are not specific for the different ureaplasma serotypes. ${ }^{8}$

Chlamydia antigen. Chl. trachomatis strain GF 726 was obtained initially from Dr Wanda Wenman, Department of Pediatrics, University of Manitoba, Winnipeg and subsequently from Dr W Bowie, Division of Infectious Diseases, Department of Medicine, University of British Columbia. The GF 726 strain was propagated in McCoy cells and cultured in minimum essential medium (MEM) supplemented with $10 \%$ fetal calf serum for three days. After removal of the medium $0.5 \mathrm{ml}$ sucrosephosphate was added and the cells frozen and thawed three times to give the antigen preparation. Similarly treated uninoculated McCoy cells formed the control antigen which was used in early experients until its failure to stimulate lymphocytes was shown. Previous studies have shown that the lymphocyte responses were similar for the GF 726 strain as for the 595/8 strain. ${ }^{8}$ Storage of the antigen was at $-70^{\circ} \mathrm{C}$.

Enterobacteriaceae antigens. Pilot experiments at the beginning of the investigation established that synovial lymphocyte responses were the same for crude Enterobacteriaceae antigens made in the two following ways. Organisms cultured in trypticase soy broth were centrifuged and the deposit washed once in PBS before resuspension in distilled water, with 
three freeze-thaw cycles and the addition of penicillin 400 units and streptomycin $400 \mathrm{mg} / \mathrm{l}$; such antigenic preparations were labelled 'total' antigens. A second antigenic preparation labelled ' $\mathrm{O}$ ' antigen was derived from trypticase soy slant cultures; the deposit of washed organisms obtained after centrifugation was subsequently boiled for $150 \mathrm{~min} .{ }^{11}$ In the final standardisation of the method the use of the boiled antigen was selected, and the preparative method was kept constant. Trypticase soy agar was prepared in $7 \mathrm{ml}$ volumes and solidified at a standard angle giving a constant growth surface. Recloned Enterobacteriaceae strains on blood agar were seeded onto the slants and incubated at $37^{\circ} \mathrm{C}$ for $24 \mathrm{~h}$. The growth of each slant was scraped off, washed once in PBS, and then resuspended in $1 \mathrm{ml}$ of distilled water. After boiling for $150 \mathrm{~min}$ an additional $1 \mathrm{ml}$ of RPMI medium containing penicillin $200 \mathrm{U}$ and streptomycin $200 \mu \mathrm{g}$ was added, and the antigenic preparation was stored at $-70^{\circ} \mathrm{C}$. Batches of antigen were made from multiple slants so that a single batch would last approximately one year.

Strains of Salm. typhi, Salm. enteritidis, and Salm. typhimurium were all isolated locally. The enteritidis strain was from a patient with reactive arthritis, because it was thought possible that arthritogenic properties might be related to particular strains with geographical localisation, and we had recent experience with salmonella arthritis in British Columbia. ${ }^{12}$ Multiple tests with antigens derived from these three strains showed that they gave similar lymphocyte stimulating results. The strain of Sh. flexneri employed for these studies was isolated from a local patient who subsequently developed postdysenteric arthritis. The Escherichia coli antigen was derived from a viable strain of ATCC 25922 obtained from Difco.

Campylobacter antigen. The antigenic preparation, derived from a locally isolated strain of $C$. jejuni, was made by the same procedure as that for the Enterobacteriaceae $O$ antigens, with the exception that the slant cultures were incubated at $42^{\circ} \mathrm{C}$ for $48 \mathrm{~h}$.

Viral antigens. These antigens employed for the studies in patients Nos 5 and 9 were obtained commercially from Microbiological Associates or Flow Laboratories and are described in detail elsewhere. $^{13}$

Mycoplasma hominis antigen. Initially in the study an $M$. hominis antigen was employed. This was a washed broth deposit of organisms grown in the same broth as that for ureaplasmas, with the difference that $1 \%$ arginine was added instead of urea.
STASTISTICAL METHODS

The accuracy and variability of the method were determined by calculating the coefficient of variaition of the $\left[{ }^{3} \mathrm{H}\right]$ thymidine triplicate uptake counts There were differences between cases and the accuracy varied between $\pm 10 \%$ and $\pm 25 \%$.

The analyses in Tables 3 and 4 were based on the logarithms of the cpm ratios of the $\left[{ }^{3} \mathrm{H}\right]$ thymiding uptakes under stimulated versus unstimulated cons ditions for the synovial and peripheral blood re sponses respectively. Logarithms were used to stabilise the variances. Paired $t$ tests were the performed and the results shown in the tables.

\section{Results}

The reproducibility of these observations on syn $\overrightarrow{\vec{\theta}}$ vial lymphocyte responses is illustrated in Fig. IJ which shows the stimulation indexes from synoviat aspiration on four successive weeks in patient No $\$$ with sexually transmitted reactive arthritis. On the first occasion a stimulation index of over 200 to ureaplasma antigen contrasted with a stimulatiog index of 33 to phytohaemagglutinin (PHA) an negligible indexes to the ureaplasma broth controto an M. hominis antigen, and mumps antigen. Onghge next two aspirations the ureaplasma index remaimed above 250; although there was some increase in indexes to the other antigenic preparations, the contrast between the ureaplasma response and the other responses remained essentially the same. OA the fourth aspiration when the arthritis in the knees was subsiding all the responses were less, but th $\overrightarrow{\vec{E}}$ ureaplasma response was still three times the mumps response. Peripheral blood lymphocy responses were first studied at the time of the thin knee joint aspiration, and repeated observation were made on the blood lymphocyte responses aftef the synovial effusion had resolved. The data in the figure show the consistency of both the synovial and blood responses when studied by the methoos employed in this investigation.

Evidence of reproducibility of results by the techniques used in these studies is also shown by the synovial lymphocyte data of two cases of enterit. reactive arthritis, patients Nos 1 and 4 (Table 2). 捻 patient No 1, whose arthritis followed a Salm. typhimurium enteritis, the synovial lymphocytes responded more to salmonella antigens than to other enteric antigens on both occasions. On the second occasion all the stimulation indexes were approximately twice as high, but the ratios of the responses between antigens were similar. In patient No 4, whose arthritis was diagnosed as being postdysenteric reactive arthritis, the synovial lyng phocytes responded to shigella antigen with a highe 


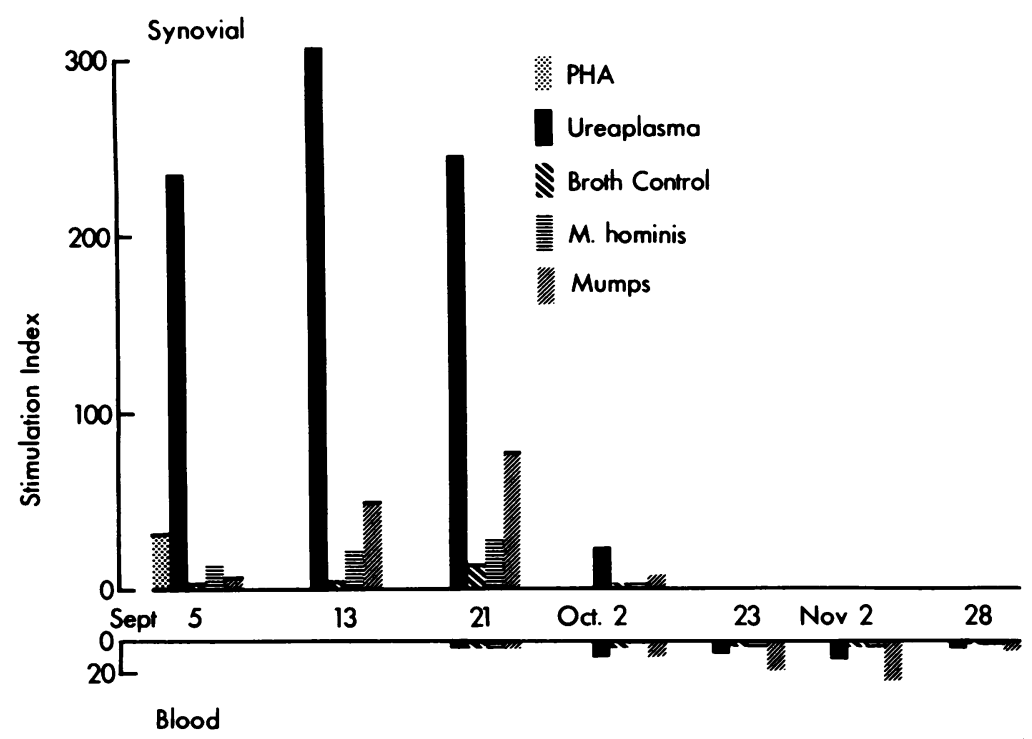

Fig. 1 Synovial and peripheral blood lymphocyte responses in patient No 9 with sexually transmitted reactive arthritis, when tested on the dates shown in the figure.

Table 2 Synovial lymphocyte responses to enteric antigens in five cases of arthritis associated with bacteriologically defined enteritis

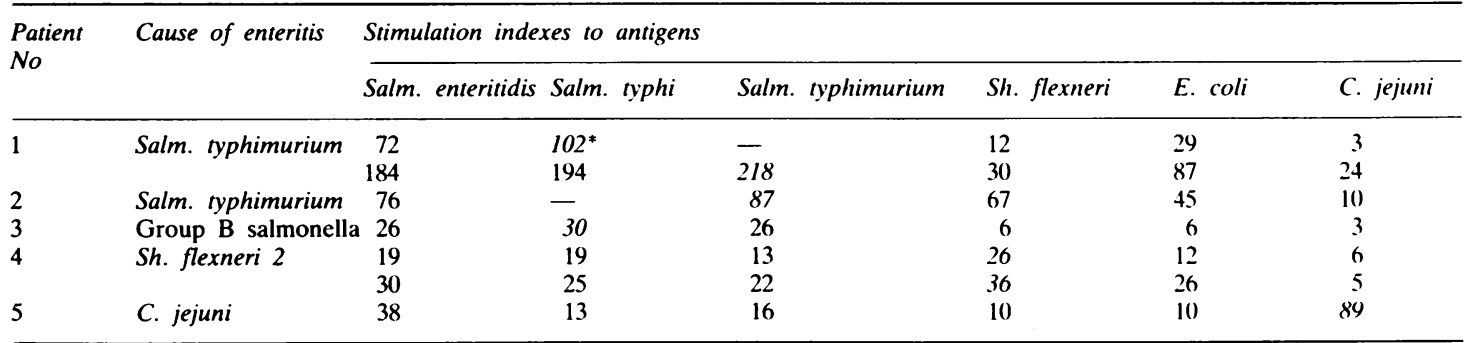

*The italic numbers indicate the antigen related to the cause of the reactive arthritis: the first three cases were of salmonella origin. case No 4 was of dysenteric origin, and No 5 of campylobacter origin.

stimulation index than to the other enteric antigens on both occasions they were tested; once again the ratios of the stimulation indexes for the individual antigens were similar in the two testings.

Table 2 shows the specificity of the synovial lymphocyte responses in cases of bacteriologically defined enteric reactive arthritis. Two patients (Nos 1 and 2) had Salm. typhimurium infections, and one patient (No 3) had salmonella group B infection. Synovial lymphocytes of the above three cases responded highly to salmonella antigens but could not differentiate between the three salmonella species: namely, Salm. typhimurium in group B, and Salm. typhi and Salm. enteritidis which belong to group D serotypes. However, synovial lymphocyte responses in the five cases of bacteriologically defined enteric reactive arthritis could differentiate between the four genuses, namely Salmonella, Shigella, Escherichia, and Campylobacter, as shown in Table 2. In three cases (Nos 1,2, and 3) of defined salmonella enteritis synovial lymphocytes showed the highest response to salmonella antigens in comparison with the other three enteric antigens. In case No 4, in which the enteritis was serologically defined with a serum antibody titre of 1/64 to Sh. flexneri 2, synovial lymphocytes responded most highly to shigella antigen. Likewise, in case No 5 . which had defined $C$. jejuni enteritis, synovial lymphocytes responded most highly to campylobacter antigen in comparison with the other three enteric antigens.

The specificity of the reactions is shown in greater detail for the campylobacter case in Fig. 2. The stimulation index of 89 against campylobacter anti- 


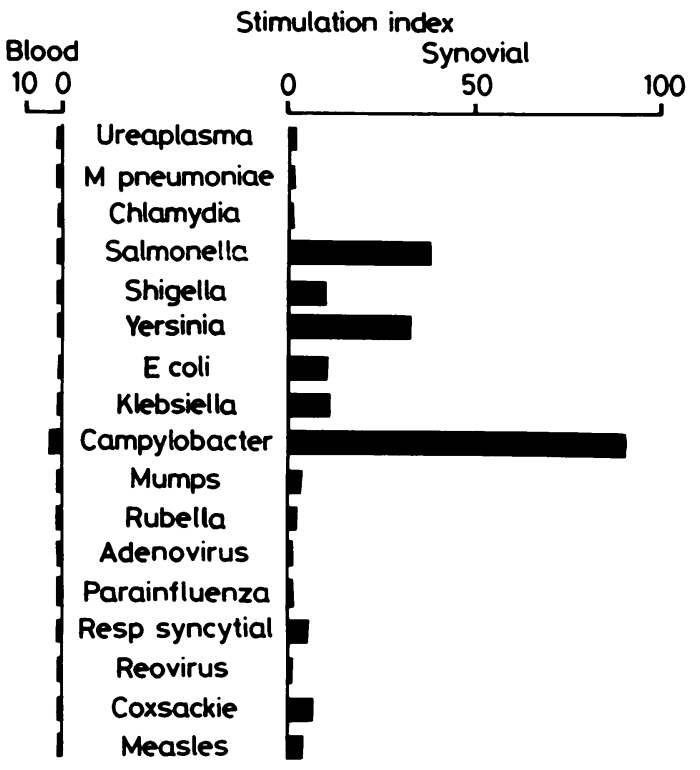

Fig. 2 Synovial and peripheral blood lymphocyte responses in patient No 5 with reactive arthritis complicating campylobacter enteritis. gen contrasted with a lower degree of stimulatio $(10-38)$ to Enterobacteriaceae antigens. Minimal of negligible stimulation was present against eight viral antigens, and also ureaplasma, chlamydia, an雨 $M$. pneumoniae antigens. More recently a candide antigen has been included in the battery of tes antigens, this was not done at the time these case were studied, but this added 'control' antigen ha $\bar{\Phi}$ not altered the interpretation of the results of the subsequently studied cases.

Figs 3,4 , and 5 give the stimulation indexe $\vec{P}$ obtained from both synovial and peripheral blood lymphocytes against ureaplasma and chlamydiक antigens and also the enteric antigen which gave the maximal response. In Fig. 3 the eight enteric cases showed that the responses of the synovial lymphot cytes were uniformly greater to enteric antigens that to either ureaplasma or chlamydia. There is a strong suggestion that there was some associated stimula $=$ tion to chlamydia antigen, but this was always les? than to the enteric antigen. The low or negligibleperipheral blood lymphocyte responses to enterie antigens are evident.

In Fig. 4 equivalent data are barographed for the 12 cases of sexually transmitted reactive arthritis case No 9 is included although responses to chlanes

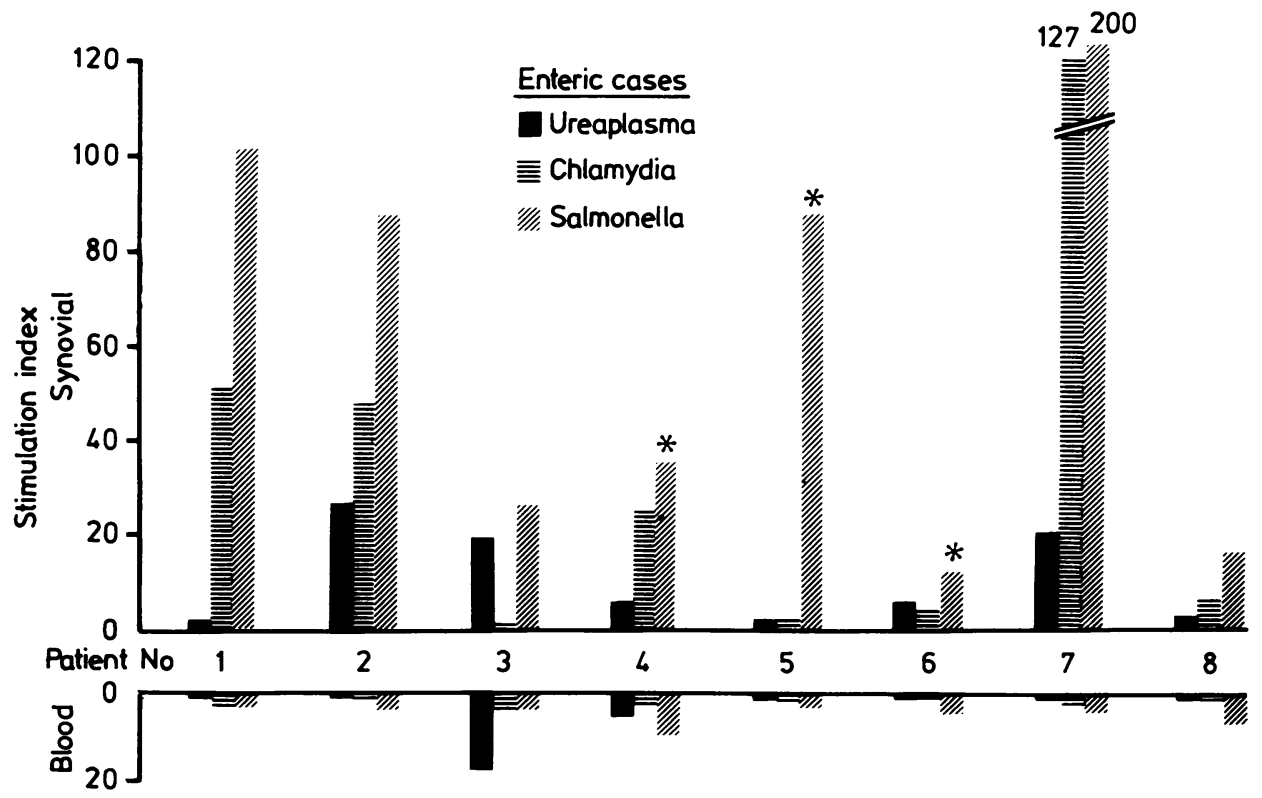

Fig. 3 Synovial and peripheral blood lymphocyte responses to ureaplasma, chlamydia, and salmonella antigens in eight cases of enteric reactive arthritis. In patients Nos 4 and $6\left(^{*}\right)$ the maximal response was to shigella antigen and in patient No $\left(^{*}\right)$ the maximal response was to campylobacter antigen. 


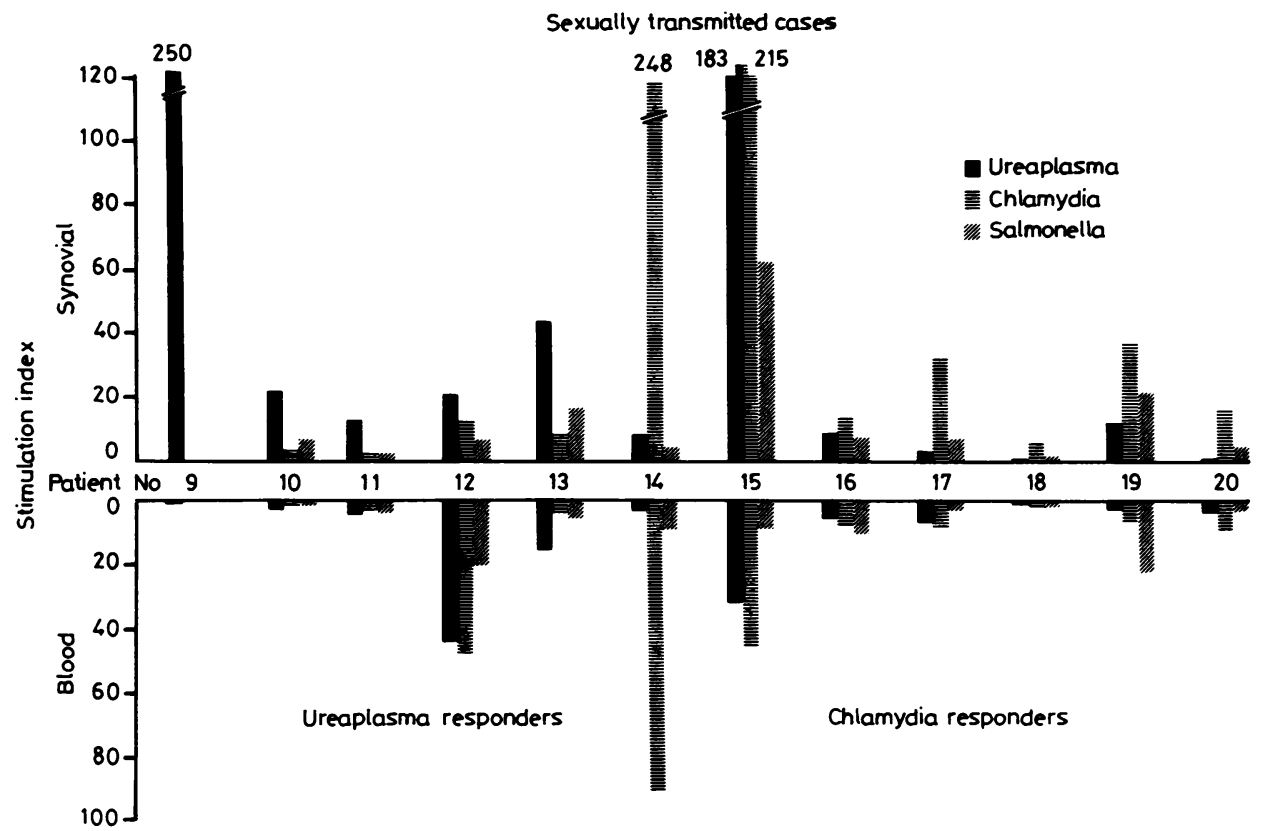

Fig. 4 Synovial and peripheral blood lymphocyte responses to ureaplasma, chlamydia, and salmonella antigens in 12 cases of sexually transmitted reactive arthritis.

dia and enteric antigens were not tested. Four of the 12 patients had maximal responses to ureaplasma antigen and seven to chlamydia antigen. In cases 10 to 20 the responses to the enteric antigens were less. Comparison of the synovial with the peripheral blood lymphocyte responses showed that the differences were not as marked as in the enteric cases. Three of the 12 had stimulation indexes of two from peripheral blood lymphocytes, a negative response. Five cases had stimulation indexes of 4-9, low responses. Four cases had indexes between 15 and 90 , strong responses, and in one of these (patient No 12) the peripheral blood response was higher than the synovial response. Two of the strong response group, patients Nos 12 and 14, had previous episodes of sexually transmitted reactive arthritis within a year of the studied episodes and this may have been a related factor. Whereas patients can be expected to have only one episode of salmonellosis, shigellosis, or campylobacter infection, recurrent non-gonococcal urethritis and recurrent sexually transmitted arthritis are common, and this may have contributed to the greater responsiveness of the peripheral blood lymphocytes in the sexually transmitted group. Duration of the particular episode before the time of study did not seem to be a factor, as patients Nos 18,19 , and 20 had long intervals with low responses, and patient No 15 had a high

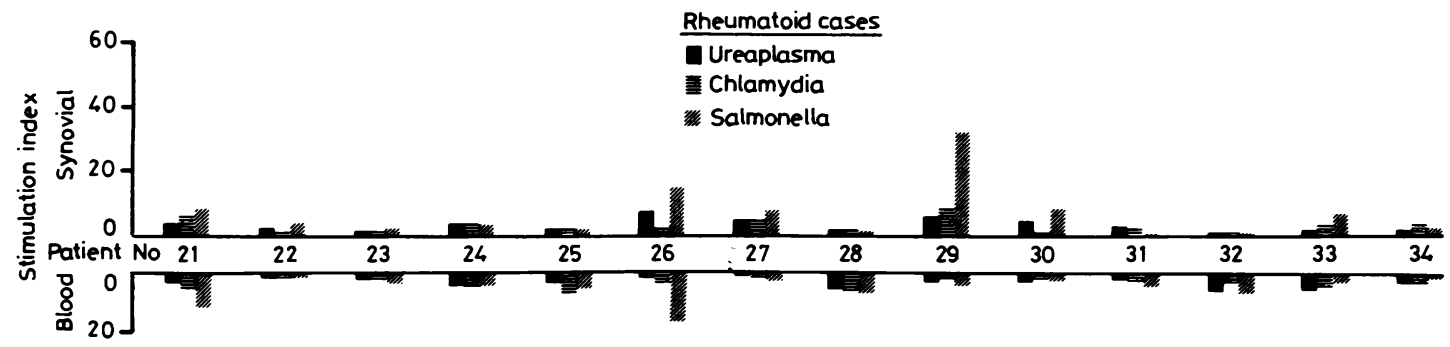

Fig. 5 Synovial and peripheral blood lymphocyte responses to ureaplasma, chlamydia and salmonella antigens in 14 cases of rheumatoid arthritis. 
peripheral blood response with a short duration of disease. The reliability of histories of the presence or absence of previous non-gonococcal urethritis in these patients is most uncertain.

The results of stimulating synovial and peripheral blood lymphocytes from patients with rheumatoid arthritis are shown in Fig. 5. The responses to these ureaplasma, chlamydia, and enteric antigens were minimal. One patient, No 29, had a synovial stimulation index of 37 to salmonella antigen. This may have been related to the fact that he was in the
Far East during World War II and would have bee immunised with $T A B$ vaccine. He was subsequentl a travel agent and had been given booster TAB. vaccine about five years before the present studies

Tables 3 and 4 give the $\left[{ }^{3} \mathrm{H}\right]$ thymidine uptake counts and stimulation indexes of the response to the relevant antigen by both synovial and peripherat blood lymphocytes in the eight cases of enteri而 reactive arthritis and the 12 cases of sexuall transmitted reactive arthritis. The $\left[{ }^{3} \mathrm{H}\right]$ thymidine uptake by unstimulated synovial and blood lymphö

Table 3 Comparison of synovial and peripheral blood lymphocyte responses in eight patients with enteric reactive arthritis

\begin{tabular}{|c|c|c|c|c|c|c|c|}
\hline \multirow[t]{4}{*}{ Patient No } & \multirow[t]{4}{*}{ Antigen ${ }^{*}$} & \multicolumn{6}{|c|}{$l^{3} H / T h y m i d i n e$ uptake } \\
\hline & & \multicolumn{2}{|c|}{ Unstimulated cells } & \multicolumn{4}{|c|}{ Stimulated with relevant enteric antigen } \\
\hline & & \multicolumn{2}{|c|}{ Counts per min } & \multicolumn{2}{|c|}{ Counts per min } & \multicolumn{2}{|c|}{ Stimulation index } \\
\hline & & Synovial & Blood & Synovial & Blood & Synovial & Blood \\
\hline 1 & $\mathrm{~S}$ & 1166 & 346 & 118560 & 1177 & 102 & 3 \\
\hline 2 & $\mathrm{~S}$ & 1293 & 1305 & 112514 & 5228 & 87 & 4 \\
\hline 3 & $\mathrm{~S}$ & 3108 & 1192 & 80981 & 3148 & 26 & 3 \\
\hline 4 & $\mathrm{D}$ & 3751 & 202 & 135020 & 1757 & 36 & 9 \\
\hline 5 & $\mathrm{C}$ & 634 & 1240 & 56541 & 3203 & 89 & \\
\hline 6 & D & 3299 & 2223 & 51253 & 3675 & 16 & \\
\hline 7 & $\mathrm{~S}$ & 123 & 2472 & 24630 & 7880 & 200 & 30 \\
\hline 8 & $\mathrm{~S}$ & 2226 & 632 & 38027 & 5087 & 17 & 8 बे \\
\hline
\end{tabular}

Table 4 Comparison of synovial and peripheral blood lymphocyte responses in 12 patients with sexually transmitte reactive arthritis

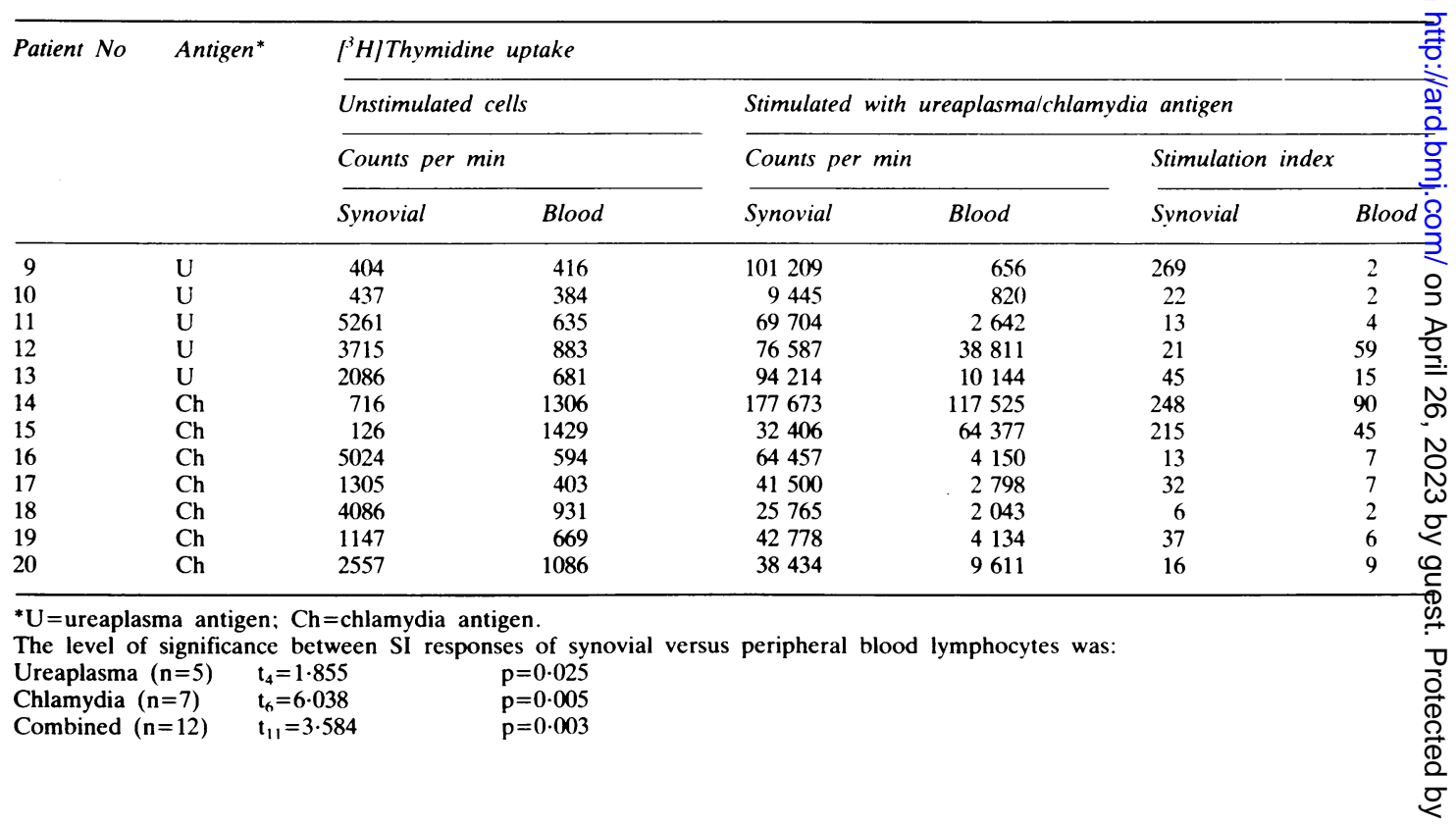


cytes is included in the tables. The statistical level of significance in the comparison between the synovial and peripheral blood stimulation indexes is shown below the tables. The synovial responses greatly and uniformly exceeded the peripheral blood responses in the enteric cases. Thus despite high stimulation indexes for synovial lymphocytes, the peripheral blood lymphocytes gave negligible indexes in six of the eight cases and only low level stimulation in two, with indexes of 8 and 9 (Table 3).

When synovial and peripheral blood lymphocyte responses in the 12 sexually transmitted cases were compared the greater responses of the synovial lymphocytes were statistically significant, as shown by Table 4 . Four of the 12 cases did have strong responses from blood lymphocytes, and in one case (No 12) the blood index was higher than the synovial index. However, the synovial lymphocytes in these four cases also gave high stimulation indexes, and the $\left[{ }^{3} \mathrm{H}\right]$ thymidine uptake was higher for synovial lymphocytes in three of the four. In four of the 12 cases the blood responses were negligible.

\section{Discussion}

The above findings show that the synovial lymphocytes may indicate the cause of reactive arthritis when peripheral blood lymphocytes give no such indication. This observation may be important for a variety of reasons.

First, the recognition of reactive arthritis is often difficult because diarrhoea or urethritis may be minimal and overlooked by the patient. Moreover, it is frequently not possible to establish the aetiology of the intestinal or genital infection because the symptoms and signs have subsided by the time arthritis develops and appropriate cultures are not available. Alternatively, antibiotics have been given before the intestinal or genitourinary diagnosis has been established. The study of synovial lymphocyte responses may therefore be of diagnostic help.

Secondly, the relationships of chlamydias and ureaplasmas to sexually transmitted reactive arthritis is still debated. The present findings add support to the view that these organisms are aetiologically related to the arthritis. The lymphocytes at the site of the localised arthritis have the capacity to recognise these antigens when blood lymphocytes do not, moreover, this recognition is specific and not a manifestation of local lymphocyte over-reactivity.

Thirdly, the findings are not restricted to the bacterial, chlamydia, and ureaplasma antigens discussed in this report. Two cases, one of rheumatoid arthritis and one of recurrent knee arthritis, have been reported in which synovial responses to rubella antigen were associated with the isolation of rubella virus from the patient. ${ }^{14} \mathrm{~A}$ third patient with polyarthritis of 10 years' duration is currently under investigation with similar findings, a consistent synovial lymphocyte response to rubella with the isolation of the virus from the joint. ${ }^{15}$ In none of these three patients did the peripheral blood lymphocytes respond to rubella antigen. Therefore, in the study of diseases of unknown aetiology, in which immunological reactions are a pathogenetic feature, it is possible that interest should be focused on lymphocytes obtained from the site of local disease in preference to the study of peripheral blood lymphocytes.

Fourthly, the existence of marked responses to antigenic stimulation by synovial lymphocytes when peripheral blood lymphocytes are unreactive raises basic questions as to the reasons for this discrepancy. The synovial lymphocytes are clearly capable of immunological functions which are not shared by lymphocytes circulating in the peripheral blood at the same time. It has not been possible in this laboratory up to the present to define the class of lymphocytes in the studied preparations.

Fifthly, an associated study ${ }^{16}$ of patients with arthritis confined to knee joints has shown that approximately a third of patients have synovial lymphocytes that will respond to chlamydia/ ureaplasma antigen and another third will have synovial lymphocyte responses to the enteric antigens employed in this study. In addition, the chlamydia/ureaplasma responders are HLA distinct from the enteric antigen responders; the former have more HLA-B44 and HLA-DR7 or 8 , whereas the latter have more A1 and DR5. The synovial lymphocyte therefore has the capability of defining immunological responses under genetic control to specific microbiological agents. Moreover, the clinical handling of patients with persistent and recurrent arthritis confined to knee joints is a common rheumatological problem and one that has yielded, up to the present, no clues as to cause; the synovial lymphocyte now appears to provide new research approaches.

It is hoped that the present report will encourage interest in the responses of lymphocytes obtained at the site of local disease to microbiological agents.

This work was supported by the Arthritis Society of Canada and the British Columbia Health Care Research Foundation.

\footnotetext{
References

1 Paronen I. Reiter's discase. A study of 344 cases observed in Finland. Acta Med Scand 1948: Suppl 212.

2 Berglof F E. Arthritis and intestinal infection. Acta Rheumatol Scand 1963: 9: 1449.

3 Ahvonen P. Sievers K. Aho K. Arthritis associated with
} 
Yersinia enterocolitica infection. Acta Rheumatol Scand 1969: 15: $232-53$

4 Urman J D. Zurier R B. Rothfield N F. Reiter's syndrome associated with campylobacter fetus infection. Ann Intern Med 1977; 86: 444-5.

5 Bowie W R. Nongonococcal urethritis. Urol Clin North Am 1984; 11: 55-64.

6 Ford D K, da Roza D M, Shah P, Wenman W. Cell-mediated immune responses of synovial mononuclear cells in Reiter's syndrome against ureaplasmal and chlamydial antigens. $J$ Rheumatol 1980; 7: 751-5.

7 Ford D K, da Roza D M. Shah P. Cell-mediated immune responses of synovial mononuclear cells to sexually transmitted. enteric and mumps antigens in patients with Reiter's syndrome. rheumatoid arthritis and ankylosing spondylitis. $J$ Rheumatol 1981: 8: 220-32

8 Ford D K, da Roza D M. Schulzer M. The specificity of synovial mononuclear cell responses to microbiological antigens in patients with arthritis. J Rheumatol 1982: 9: 561-7.

9 Sigal L H. Steere A C. Dwyer J M. Evolution of cellular reactivity to the Lyme spirochete: concentration in joint fluid. Arthritis Rheum 1984; 27: S36.

10 Shepard M C. Lunceford C D. Ford D K. et al. Ureaplasma urealyticum gen. nov. sp. nov.: Proposed nomeclature for human T (T-strain) mycoplasmas. Int J Sistem Bacteriol 1974:Э
24: 160 -71.

11 Cruickshank R. Duguid J P. Marmion B P. Swain R H A क Medical microbiology. 12th ed. Edinburgh and London $\Rightarrow$ Churchill Livingstone. 1975: 408.

12 Stein H B. Abdullah A. Robinson H S. Ford D K. Salmonella reactive arthritis in British Columbia. Arthritis Rheum 1980: 23듬 206-10.

13 Ford D K. da Roza D M. Observations on the responses of synovial lymphocytes to viral antigens in rheumatoid arthriti̊ and Reiter's syndrome. J Rheumatol 1983; 10: 643-6.

14 Ford D K. da Roza D M. Reid G D. Chantler J K. Tingle A J Synovial mononuclear cell responses to rubella antigen ir $P$ rheumatoid arthritis and unexplained persistent knee arthritis. Rheumatol 1982; 9: 42()-3.

15 Chantler J K, da Roza D M. Bonnie M E, Reid G D. Ford D K Sequential studies on synovial lymphocyte stimulation bo rubella antigen. and rubella virus isolation in an adult with persistent arthritis. Ann Rheum Dis 1985: 44: 56+8.

16 Ford D K. da Roza D M. Ward R H. Arthritis confined to knec joints: synovial lymphocyte responses to microbial antigens correlate with distribution of HLA. Arthritis Rheum 1984: 27? $1157-64$

\section{Book review}

Rheumatoid Arthritis: Helping Yourself. A booklet produced by the Departments of Occupational Therapy, and Health Education and Health Promotion of Doncaster Health Authority with the collaboration of various others. 1985. Available from the Department of Health Education and Health Promotion, Alverley House, Springwell Lane, Balby, Doncaster, South Yorks. $£ 3.51$ for five bookets - the more that are bought the cheaper the rate.

The 'right to know' debate is likely to continue, and the legal implications of uninformed consent may grow more worrying to doctors. Hence literature written for patients is to be welcomed, especially if it is as lucid and sensible as in this booklet.

As its title suggests the aim is pragmatic. Sections cover general advice, kitchen work, general living, dressing, and personal hygiene. The excellent illustrations drawn by Steve Hurst and Paul Thorpe do, by themselves, make it a worthwhile addition to other practical booklets for patients. The packaging of articles today often results in almost unsurmountable difficulties in opening them, evem for those with normal hands - and impossible for those with rheumatoid ones; the 'Slitapac' is the answer fo 5 packets and the 'twister' or 'strongboy' for jars. Health housewives might also profit by a look at this booket?

Some information about rheumatoid arthritis (RA) is provided: about $x$-rays, the difference between osteoarthr? tis and RA, how common is RA?, why do joints go out of shape?, and other questions, for example, what causes rheumatoid nodules?

I liked the approach to the patient for it is neithe condescending nor paternalistic. The introduction states 'you are a member of a team', and the members of tha? team from family doctor to appliance officer, occupationad therapist, and chiropodist (altogether 10) are listed.

Just one caveat: the booklet might strike terror into some patients who have mild or early disease, since the picture on the cover shows a woman holding armfuls $\mathbb{P}$ gadgets. The booklet is intended for those who ar零 handicapped in some way or crippled; but no doubr precautions are taken in clinics and elsewhere to preven the wrong patient getting the wrong booklet.

CLIFFORD HAWKINS 